\title{
3 Research Square

\section{The application of scrotoscope-assisted minimally invasive excision for epididymal mass: an initial report}

\section{Chuying Qin}

Second Xiangya Hospital of Central South University

Jinrui Yang

Second Xiangya Hospital of Central South University

Ruochen Zhang

Fujian Provincial Hospital

Yaojing Yang

Fujian Provincial Hospital

\section{Wanghai Cai}

Fujian Provincial Hospital

Tao Li

Fujian Provincial Hospital

Qingguo Zhu

Fujian Provincial Hospital

\section{Liefu Ye}

Fujian Provincial Hospital

Yunliang Gao ( $\nabla$ yunliang.gao@csu.edu.cn )

Second Xiangya Hospital of Central South University

\section{Yongbao Wei}

Fujian Provincial Hospital

\section{Research Article}

Keywords: Epididymal mass, minimally invasive, open excision, scrotoscope

Posted Date: September 7th, 2021

DOI: https://doi.org/10.21203/rs.3.rs-858240/v1

License: (c) (i) This work is licensed under a Creative Commons Attribution 4.0 International License.

Read Full License 
Version of Record: A version of this preprint was published at Frontiers in Surgery on February 24th, 2022. See the published version at https://doi.org/10.3389/fsurg.2022.804803. 


\section{Abstract \\ Background}

Traditional open excision of epididymal mass is a non-minimal invasive treatment and brings relatively more postoperative discomfort and complications. To solve this problem, we apply scrotoscope to treat epididymal mass and compare the middle-term efficacy and safety results between scrotoscope-assisted (SA) minimally invasive excision and traditional open excision (OE) for the treatment of epididymal mass.

\section{Methods}

A total of 253 males with surgery excision of epididymal mass from 2012 to 2018 were included in this retrospective study. The primary outcomes included general information, intraoperative data and postoperative data.

\section{Results}

174 patients underwent SA and other 79 underwent OE. Demographic data was similar between the two groups. Compared with OE surgery, SA could significantly shorten operating time $(19.4 \pm 4.1$ vs $53.8 \pm$ 12.9 minutes), reduce blood loss ( $5.3 \pm 1.5$ vs $21.3 \pm 5.6 \mathrm{~mL})$ and downsize the operative incision $(1.5 \pm$ $0.3 \mathrm{~cm}$ vs $4.5 \pm 0.8 \mathrm{~cm}$ ). Additionally, postoperative complications were significantly less occurred in SA group than those in OE (15.5\% vs $21.5 \%)$. Patients in SA group had a significant higher overall satisfaction score $(94.8 \pm 3.7$ vs $91.7 \pm 4.9)$ than that in OE group.

\section{Conclusion}

SA is emerging as a novel and effective option with promising perspectives for epididymal mass therapy.

\section{Introduction}

Epididymal mass is recognized as a common disorder in male population. It appears to be a diagnostic dilemma and the most prominent types are mass-forming epididymitis, ${ }^{1,2}$ epididymal cyst, ${ }^{3}$ epididymal sperm granuloma, ${ }^{4,5}$ epididymal tuberculosis and so on. Primary tumors of the epididymis origin are rarely occurred, accounting for about $2.5 \%$ of male genital tumors ${ }^{6}$ and at most $0.03 \%$ of all male cancers. ${ }^{7}$ Adenomatoid tumor is the most common type of epididymal tumors. Epididymal masses are almost always benign without specific treatment. However, parts of patients are admitted to the hospital due to different degrees of scrotal symptoms such as scrotal distention, chronic pain, and tenderness. When an epididymal mass does not benefit from conservative treatment, surgical intervention appears to be considered. 
For the surgical treatment of epididymal mass, the traditional open excision of mass (OE) is one of the main choices. However, $\mathrm{OE}$ offers a non-minimal invasive treatment to scrotum and brings relatively more postoperative discomfort and complications (hematomas, infection, etc.). ${ }^{8,9}$ Firstly described by Gerris and Shafik, ${ }^{10,11}$ the scrotoscope has been found to be a minimally invasive and less complicated operation for the diagnosis and treatment of scrotal diseases. As described previously, we have successfully applied scrotoscope to manage different scrotal diseases including epididymal cyst, adult testicular hydrocele, testicular rupture, testicular torsion, and all achieved satisfactory results. ${ }^{12-16}$ In order to further improve surgical outcomes, this study was carried out for the evaluation of the feasibility and efficacy of the scrotoscope-assisted (SA) excision of epididymal masses.

\section{Results}

\section{General Information}

A total of 253 patients with epididymal masses were enrolled in this retrospective study, 174 underwent SA and 79 underwent OE. The mean age was $47 \pm 12.8$ (22-80) years in SA and $48 \pm 14.9$ (22-80) years in $\mathrm{OE}$, respectively. The mean follow-up time was $20.8 \pm 8.2$ months in SA vs $19.4 \pm 8.6$ months in OE. Table 1 presents a summary of patients' characteristics. Two groups showed no significant differences in terms of age, time since onset, follow-up period, mass size (maximal diameter) on ultrasound and the side of treatment.

Table 1. Demographic Characteristics

There was no significant difference found in demographic characteristics of both groups, including the age, duration of disease, mass size, and follow-up times, preoperative ultrasound result, and mass side (all $p>0.05$ ). Abbreviations: $\mathrm{OE}$, open excision; $\mathrm{SA}$, scrotoscope-assisted excision.

\section{Intraoperative Data}

All patients underwent surgery successfully. The mean operating time in SA was significant shorter than OE (19.4 \pm 4.1 vs $53.8 \pm 12.9$ minutes). The blood loss in SA was significantly less than $O E(5.3 \pm 1.5$ vs $21.3 \pm 5.6 \mathrm{~mL})$. The mean incision size in SA was significantly shorter than that in $\mathrm{OE}(1.5 \pm 0.3 \mathrm{vs} 4.5 \pm 0.8$ $\mathrm{cm})$.

\section{Postoperative Data}

Patients in SA group had a significant less frequency of dressing changes ( $2.9 \pm 1.3$ vs $4.4 \pm 1.7$ times) and a significant shorter length of postoperative hospital stay ( $4.1 \pm 0.9$ vs $5.0 \pm 1.5$ days) when compared with 


\begin{tabular}{|llll|}
\hline & $\mathrm{SA}(\mathrm{n}=174)$ & $\mathrm{OE}(\mathrm{n}=79)$ & P value \\
\hline Age (year) & $47.9 \pm 12.8$ & $48.0 \pm 14.9$ & 0.962 \\
\hline Duration of disease(year) & $2.5 \pm .6$ & $2.5 \pm 0.6$ & 0.403 \\
\hline Maximum diameter (cm) & $3.2 \pm 1.1$ & $3.1 \pm 1.1$ & 0.608 \\
\hline Follow-up time (year) & $20.8 \pm 8.3$ & $19.4 \pm 8.6$ & 0.219 \\
\hline Mass side & & & \\
\hline Left & 80 & 40 & 0.492 \\
\hline Right & 94 & 39 & \\
\hline Location & & & \\
\hline Caput & 36 & 15 & 0.937 \\
\hline Corpus & 53 & 26 & \\
\hline Cauda & 42 & 17 & \\
\hline Diffuse & 43 & 21 & \\
\hline
\end{tabular}

OE group (Figure 2). A significant higher overall satisfaction score was found in SA group rather than in OE group (94.8 \pm 3.7 vs $91.7 \pm 4.9)$.

No significant difference was reported for the total incidence of complications as well as complication Grades, which is 27 (15.5\%) in SA group and 17 (21.5\%) in OE group (Grade I-III) (Table 2.). Among them, almost equal incidence of postoperative recurrence was observed, which were 5 (2.9\%) in SA and $2(2.5 \%)$ cases in OE. SA were reported that $19(10.9 \%)$ cases occurred scrotal edema while in $\mathrm{OE}$ was $0(0 \%)$. However, the incidence of scrotal hematoma in SA was less than OE, which were $3(1.7 \%)$ vs $10(12.7 \%)$ cases. Less case suffered incision discomfort in SA, which were $5(2.8 \%)$ cases in SA vs $5(6.3 \%)$ cases in OE. No testicular atrophy (0\%) occurred in SA but $1(1.3 \%)$ case in $\mathrm{OE}$. No testicular, spermatic cord injury or secondary hydrocele occurred. Except for cases with slight scrotal discomfort after operation, all the other patients' preoperative symptoms were significantly relieved.

Table 2. Complications and classification

The incidence of SA complications was less than $\mathrm{OE}$, the symptoms of complications were relatively milder in SA. Abbreviations: OE, open excision; SA, scrotoscope-assisted excision.

\section{Discussion}

Our study firstly highlighted the feasibility of scrotoscopy in the treatment of epididymal masses. As shown by our results, SA demonstrated a significant superiority over traditional OE for the treatment of epididymal mass.

Epididymal masses are common seen in urological clinic. Typically, the patient seeks medical consultation due to an incidentally palpated, painless scrotal mass. Epididymal masses still seem to be a diagnostic and therapeutic dilemma, despite most of them being benign lesions. Primary epididymal tumor are rarely occurred and most common of them were adenomatoid tumors. ${ }^{7}$ Solid mass can be touched with different sizes, often originating in the epididymal caput or cauda. Scrotal sonography is a 


\begin{tabular}{|c|c|c|c|c|}
\hline & & SA (\%) & $\mathrm{OE}(\%)$ & $P$ value \\
\hline \multirow[t]{2}{*}{ Total complications } & No & $147(84.5)$ & $62(78.5)$ & 0.243 \\
\hline & Yes & $27(15.5)$ & $17(21.5)$ & \\
\hline \multirow[t]{3}{*}{ Complication classification } & I & $22(12.6)$ & $12(15.2)$ & 0.099 \\
\hline & II & $5(2.9)$ & $4(5.1)$ & \\
\hline & III & $0(0)$ & $1(1.3)$ & \\
\hline \multirow[t]{3}{*}{ Relief of symptoms } & Complete & $155(89.1)$ & $66(83.5)$ & 0.134 \\
\hline & Partial & $18(10.3)$ & $10(12.7)$ & \\
\hline & None & $1(0.6)$ & $3(3.8)$ & \\
\hline \multirow[t]{2}{*}{ Recurrence } & No & $169(97.1)$ & $77(97.5)$ & 0.878 \\
\hline & Yes & $5(2.9)$ & $2(2.5)$ & \\
\hline \multirow[t]{3}{*}{ Scrotal edema } & No & $155(89.1)$ & $79(100)$ & 0.009 \\
\hline & Slight & $15(8.6)$ & $0(0)$ & \\
\hline & Severe & $4(2.3)$ & $0(0)$ & \\
\hline \multirow[t]{2}{*}{ Scrotal hematoma } & No & $171(98.3)$ & $69(87.3)$ & 0.000 \\
\hline & Yes & $3(1.7)$ & $10(12.7)$ & \\
\hline \multirow[t]{2}{*}{ Incision discomfort } & No & 169 (97.1) & $74(93.7)$ & 0.191 \\
\hline & Yes & $5(2.9)$ & $5(6.3)$ & \\
\hline \multirow[t]{2}{*}{ Testicular atrophy } & No & $174(100)$ & $78(98.7)$ & 0.137 \\
\hline & Yes & $0(0)$ & $1(1.3)$ & \\
\hline
\end{tabular}

recommended imaging technique to analyze the scrotal abnormality, and MRI is usually applied in selected patients. Combining with clinical history, sonographic findings could aid to establish a short differential diagnosis and subsequent plan of care. Currently, treatment for epididymal mass is still not established and conservative treatment is the main choice. However, the patients may be indicated for surgical excision of epididymal mass under the following conditions: failed medicine treatment with/without obvious symptoms, or suspended malignant tumor. ${ }^{19-21}$ In the past, open resection of epididymal mass or open partial epididymectomy was most commonly chosen for epididymal mass surgical treatment. ${ }^{22}$ However, open surgery had a relatively larger trauma and a higher postoperative morbidity-in particular hematoma and infection, which was not conducive to the rapid recovery of patients. ${ }^{23-25}$ It is a necessity to develop a minimally invasive approach in surgical treatment of scrotal diseases.

Firstly described by Gerris and Shafik, ${ }^{10,11}$ the scrotoscopy has been found to be a minimally invasive and less complicated operation for the diagnosis and treatment of intrascrotal diseases. In 1992, scrotoscopy was first performed by our group in Chinese population. We then expanded the application range of scrotoscopy for the management of other scrotal diseases, including testicular torsion, ${ }^{26}$ testicular rupture, ${ }^{13,15}$ testicular hydrocele, ${ }^{14,17,27}$ and some rare benign diseases of the scrotum. ${ }^{13-15}$ All 
of them have achieved favorable results. Compared with traditional open approach, scrotoscope-aided one has obvious advantages such as a shorter operation time, a smaller incision, and a faster recovery after surgery of fast, minimally invasive, rapid recovery and fewer complications. ${ }^{12}$

Consistently, we applied scrotoscopy for the treatment of epididymal masses and achieved superior results over traditional approach. In present study, SA showed significantly less operating time, less blood loss, and shorter length of incision than that in OE. Possible reasonings for these results are given below. Mean operating time in SA was less than $\mathrm{OE}$, because more time were need to achieve haemostasis in $\mathrm{OE}$. The endoscope can easily insert into the cavity of tunica vaginalis and can easily observe the spacelimited cavity, so the incision does not need to be as long as the OE. With the application of electric cutting technology, no obvious bleeding was observed during the SA process. These above supports that scrotoscope could serve as a feasible and efficient tool for the treatment of epididymal mass.

In terms of surgical safety, SA group showed less postoperative complications and a faster recovery when compared with $\mathrm{OE}$ group. According to the Clavien-Dindo grading system, all the postoperative complications in two groups were Grade I-III. One Grade III case was reported in OE group but none in SA group. Scrotal edema was the most common complication in SA, this may be related to the damage of perididymis or extra fluid infiltration into the interlayer of the scrotal wall through the incision. Generally, avoiding wall sheath damage and reducing perfusion pressure and time can effectively avoid edema. ${ }^{12,17}$ Based on our own experience, a maintained 60-80-centimeter hydraulic pressure is preferred. Scrotal hematoma, however, was the most common complication in $\mathrm{OE}$ rather than in SA, possibly due to the relative larger surgical trauma in open procedure. For SA patients, the mass could be minimally excised under scrotoscope and the bleeding site could be electrocoagulated without extruding the testicle from the scrotum, minimizing the risk of bleeding. Of note, no case of testicular atrophy occurred in SA group but one in OE group, potentially due to no extrusion of testicle and spermatic cord from the scrotal incision. Besides, SA was performed under clear direct vision to avoid severe damage. As shown in Table 2, patients with SA also lead to a significantly higher rate of symptom relief and higher score in satisfaction, suggesting that patients were highly satisfied with SA. These may be attributed by multiple advantages of SA, including less postoperative complications, less incision discomfort, less trauma, less dressing changes but a more rapid recovery. When it comes to postoperative recurrence, there was no significant difference between the two groups. SA had less frequency of dressing changes, probably due to the shorter incisions and better bleeding control.

However, certain limitations should be addressed in this research, including the retrospective nature, the limited size of the total population and lack of long-term follow-up data. Future studies with larger sample sizes should be conducted to further validate its diagnostic and therapeutic value.

In conclusion, our present study confirmed that SA was a safe and effective minimally invasive therapeutic option for epididymal mass. It has the advantages of small incision, rapid recovery and low risk of complications. It is worthy of further clinical application. 


\section{Methods}

\section{Study Population}

A retrospective analysis was performed at the Second Xiangya Hospital and Fujian Provincial Hospital from January 2002 to 2018. Study approval was obtained from the Ethics Committee of Fujian Provincial Hospital (K-2019-10-03). We confirmed that all methods were carried out in accordance with relevant guidelines and regulations. And the informed consent was obtained from all subjects and/or their legal guardian. As it is retrospective study and we are unable to provide informed consent for the same, the informed consent was waived by ethical committee of both Fujian Provincial Hospital and Second Xiangya Hospital. Epididymal mass was diagnosed by scrotum ultrasound. The patient was included under the following conditions: aged 18 to 60; diagnosed with epididymal mass; conservative treatments failed with or without obvious symptoms. All patients underwent a clinical assessment including vital signs, electrocardiography, laboratory examination (hemostasis parameters, routine hematology, liver and renal function, etc.). We developed C-reactive protein, purified protein derivative and chest radiography, urinary ultrasound or KUB+IVP, CT and/or scrotum MRI to exclude epididymis tuberculosis or malignant tumor. Cases with severe cardiopulmonary diseases or coagulation disorders were excluded.

\section{Main Surgical Procedures}

All patients received general anesthesia and operated in bladder lithotomy position with routine skin preparation. Disposable plastic incise drape was pasted in the operation area after sterilized. A 10F pediatric cystoscope was employed as scrotoscope. Isotonic crystalloid solution was suspended at a height of $60-80 \mathrm{~mm}$ as perfusate.

The SA procedure was performed as previous described. ${ }^{12,14,17}$ (Figure. 1) Briefly, a 1.0-cm incision was established on the affected side of the scrotum, then dissecting through the scrotal layer into the tunica sac. Two Allis forceps hold the whole scrotal wall. The scrotoscope was put into the tunica sac with continuous sterile saline infusion. The scrotal contents, including the testicle, epididymis, and tunica vaginalis were inspected sequentially. Concurrently, the location, appearance, size, margin of the epididymal mass were mainly observed. Then electrosurgical excision of epididymal mass was conducted in a systematic fashion, taking down gradually from the caput side to cauda side and reaching deeply to the plane between epididymis and testicle. Testicular injury should be carefully avoided. After excision, the wound got electrocoagulation to stop bleeding. The resected fragments of mass were retrieved using Ellik evacuator and sent for pathological examination. A scrotoscopy was reperformed to examine the scrotal contents to exclude any active bleeding or neglected lesion. The incision was sutured with absorbable stitches. A drainage strip was placed into the scrotum and removed after 24-48 hours.

For the patients treated by $\mathrm{OE}$, an about 4-cm anterior scrotal incision was made in the ill side. The epididymis and testicle were taken out of the incision and the epididymal mass was inspected and 
excised. Like SA, the incision was then closed, and a drainage strip was also used and removed within 2448 hours.

\section{Outcome Measures}

A descriptive analysis of patient demographics was performed, including age, time since onset, follow-up period and the characteristics of epididymal mass. Surgical details mainly included intraoperative (operating time, incision size, blood loss = (gauze weight after wiping all blood loss-dry gauze weight) $\mathrm{g} / 1.05 \mathrm{~g} / \mathrm{mL}$ ) and postoperative (frequency of dressing changes, complications, hospital stay, pathology finding) results. Postoperative complications were graded by Clavien-Dindo system. All patients completed at least one follow-up visit within 6 months after surgery. During the follow-up period, the patients completed the survey of the overall satisfaction of surgical treatment (ranged from 0 to 100 score). ${ }^{18}$

\section{Statistical Analysis}

Data were expressed respectively in terms of means \pm standard deviation (SD) or percentages. We compared continuous variables with $t$ tests and used a $p$-value of less than 0.05 as a cutoff for statistical significance. All data entry and analysis were carried out in SPSS 24.0 statistical analysis software (SPSS, Chicago, IL).

\section{Declarations}

Acknowledgements: None.

Data availability: The datasets generated for this study are available on request to the corresponding author.

Funding: Startup Fund for scientific research, Fujian Medical University \Grant number: 2019QH1154囚and high-level hospital foster grants from Fujian Provincial Hospital, Fujian province, China (2019HSJJ29).

Author's contributions: Yunliang Gao and Yongbao Wei conceived of the presented idea. Yongbao Wei developed the theory and performed the computations. Chuying Qin and Jinrui Yang verified the analytical methods. Yunliang Gao encouraged Chuying Qin to develop the manuscript and supervised the findings of this work. All authors discussed the results and contributed to the final manuscript

Disclosures[Drs. Chuying Qin, Jinrui Yang, Ruochen Zhang, Yaojing Yang, Wanghai Cai, Tao Li, Qingguo Zhu, Liefu Ye, Yunliang Gao, and Yongbao Wei have no conflicts of interest or financial ties to disclose.

\section{References}

1 Redshaw, J. D., Tran, T. L., Wallis, M. C. \& deVries, C. R. Epididymitis: a 21-year retrospective review of presentations to an outpatient urology clinic. The Journal of urology 192, 1203-1207, 
doi:10.1016/j.juro.2014.04.002 (2014).

2 Calleary, J. G., Masood, J. \& Hill, J. T. Chronic epididymitis: is epididymectomy a valid surgical treatment? International journal of andrology 32, 468-472, doi:10.1111/j.1365-2605.2008.00880.x (2009).

3 Karaman, A., Afsarlar, C. E. \& Arda, N. Epididymal cyst: not always a benign condition. International journal of urology : official journal of the Japanese Urological Association 20, 457-458, doi:10.1111/j.1442-2042.2012.03152.x (2013).

$4 \quad$ Tani, Y. et al. Epididymal sperm granuloma induced by chronic administration of 2methylimidazole in B6C3F1 mice. Toxicologic pathology 33, 313-319, doi:10.1080/01926230590922866 (2005).

5 Boorjian, S., Lipkin, M. \& Goldstein, M. The impact of obstructive interval and sperm granuloma on outcome of vasectomy reversal. The Journal of urology 171, 304-306, doi:10.1097/01.ju.0000098652.35575.85 (2004).

6 Hamm, B. Sonography of the testis and epididymis. Andrologia 26, 193-210, doi:10.1111/j.14390272.1994.tb00789.x (1994).

7 Yeung, C., Wang, K. \& Cooper, T. Why are epididymal tumours so rare? Asian journal of andrology 14, 465-475, doi:10.1038/aja.2012.20 (2012).

8 Kiddoo, D., Wollin, T. \& Mador, D. A population based assessment of complications following outpatient hydrocelectomy and spermatocelectomy. The Journal of urology 171, 746-748, doi:10.1097/01.ju.0000103636.61790.43 (2004).

9 Swartz, M., Morgan, T. \& Krieger, J. Complications of scrotal surgery for benign conditions. Urology 69, 616-619, doi:10.1016/j.urology.2007.01.004 (2007).

10 Shafik, A. The scrotoscope. A new instrument for examining the scrotal contents. British journal of urology 65, 209-210, doi:10.1111/j.1464-410x.1990.tb14702.x (1990).

11 Gerris, J., Van Camp, C., Van Neuten, J., Gentens, P. \& Van Camp, K. Scrotal endoscopy in male infertility. Lancet (London, England) 1, 1102, doi:10.1016/s0140-6736(88)91915-0 (1988).

12 Yang, J. R. et al. Comparison between Open Epididymal Cystectomy and Minimal Resection of Epididymal Cysts Using a Scrotoscope: A Clinical Trial for the Evaluation of a New Surgical Technique. Urology 85, 1510-1514, doi:10.1016/j.urology.2015.03.003 (2015).

13 Wei, Y. et al. Scrotoscopy exploration of testicular rupture: A pilot study. Medicine $\mathbf{9 8}$, e17389, doi:10.1097/md.0000000000017389 (2019). 
14 Lin, L. et al. Individualized minimally invasive treatment for adult testicular hydrocele: A pilot study. World journal of clinical cases 7, 727-733, doi:10.12998/wjcc.v7.i6.727 (2019).

15 Wang, Z. et al. Diagnosis and management of testicular rupture after blunt scrotal trauma: a literature review. International urology and nephrology 48, 1967-1976, doi:10.1007/s11255-016-1402-0 (2016).

16 Hong, H., Cai, W., Wu, J., Wu, X. \& Yang, J. Scrotoscopy and traditional open surgery shows a high degree of consistency in the diagnosis of testicular torsion: An initial report. Medicine 99, e21545 (2020).

17 Bin, Y., Yong-Bao, W., Zhuo, Y. \& Jin-Rui, Y. Minimal hydrocelectomy with the aid of scrotoscope: a ten-year experience. International braz j urol : official journal of the Brazilian Society of Urology 40, 384389, doi:10.1590/s1677-5538.Ibju.2014.03.13 (2014).

18 Yossepowitch, O., Aviv, D., Wainchwaig, L. \& Baniel, J. Testicular prostheses for testis cancer survivors: patient perspectives and predictors of long-term satisfaction. The Journal of urology 186, 2249-2252, doi:10.1016/j.juro.2011.07.075 (2011).

19 Jones, M. A., Young, R. H. \& Scully, R. E. Adenocarcinoma of the epididymis: a report of four cases and review of the literature. The American journal of surgical pathology 21, 1474-1480, doi:10.1097/00000478-199712000-00010 (1997).

20 Ganem, J. P., Jhaveri, F. M. \& Marroum, M. C. Primary adenocarcinoma of the epididymis: case report and review of the literature. Urology 52, 904-908, doi:10.1016/s0090-4295(98)00275-1 (1998).

21 Erikci, V. et al. Management of epididymal cysts in childhood. Journal of pediatric surgery 48, 2153-2156, doi:10.1016/j.jpedsurg.2013.01.058 (2013).

22 Vavallo, A. et al. Capillary hemangioma of the scrotum mimicking an epididymal tumor: case report. Archivio italiano di urologia, andrologia : organo ufficiale [di] Societa italiana di ecografia urologica e nefrologica 86, 395-396, doi:10.4081/aiua.2014.4.395 (2014).

23 Hicks, N. \& Gupta, S. Complications and risk factors in elective benign scrotal surgery. Scandinavian journal of urology 50, 468-471, doi:10.1080/21681805.2016.1204622 (2016).

24 Chalasani, V. \& Woo, H. H. Why not use a small incision to treat large hydroceles? ANZ journal of surgery 72, 594-595, doi:10.1046/j.1445-2197.2002.02469.x (2002).

25 Onol, S. Y. et al. A novel pull-through technique for the surgical management of idiopathic hydrocele. The Journal of urology 181, 1201-1205, doi:10.1016/j.juro.2008.10.166 (2009).

26 Ye, H. et al. A Minimally Invasive Method in Diagnosing Testicular Torsion: The Initial Experience of Scrotoscope. Journal of endourology 30, 704-708, doi:10.1089/end.2015.0724 (2016). 
27 Khaniya, S., Agrawal, C. S., Koirala, R., Regmi, R. \& Adhikary, S. Comparison of aspirationsclerotherapy with hydrocelectomy in the management of hydrocele: a prospective randomized study. International journal of surgery (London, England) 7, 392-395, doi:10.1016/j.ijsu.2009.07.002 (2009).

\section{Figures}

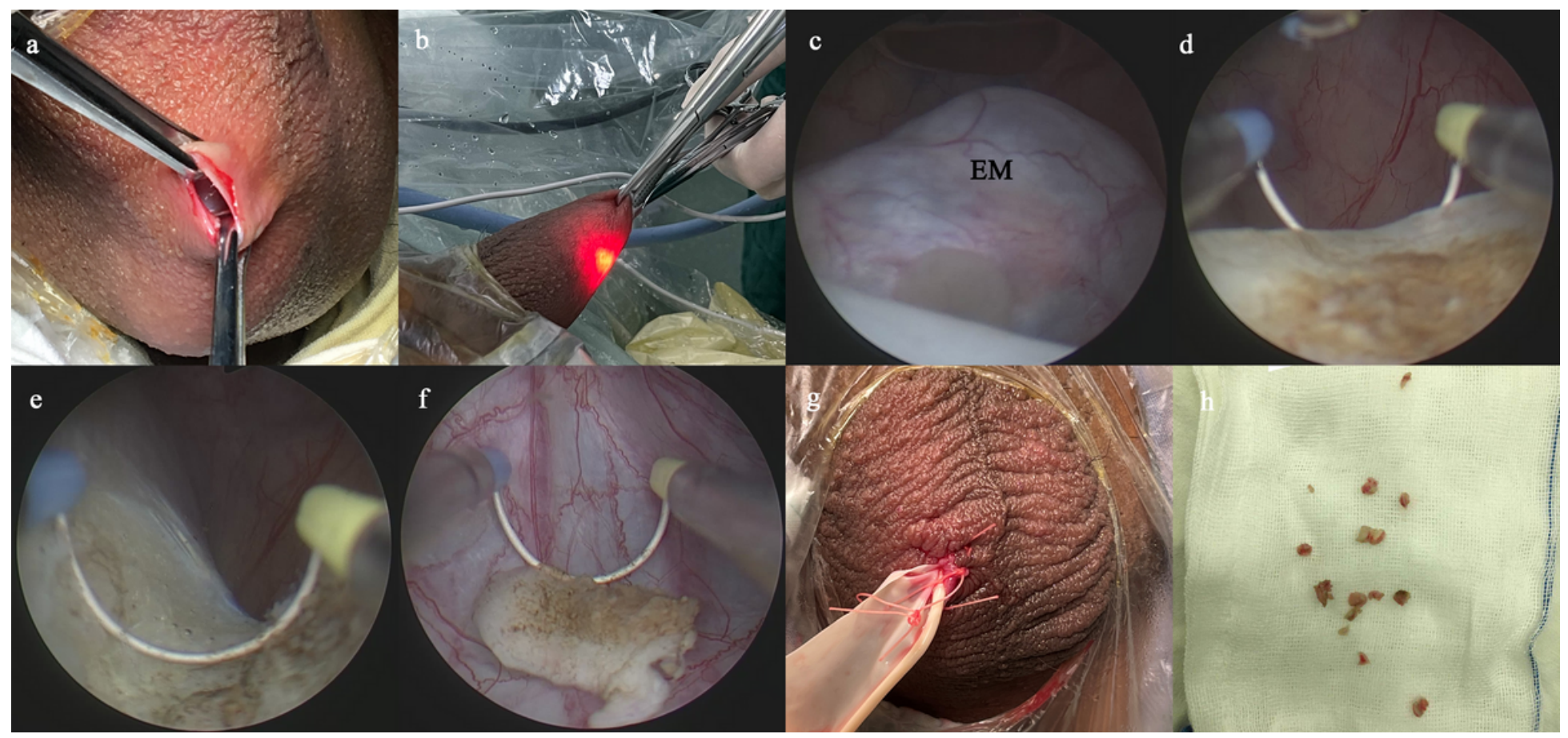

\section{Figure 1}

Main Surgical Procedures of Scrotoscope-assisted Epididymal Mass Excision. (A) A 1.0-cm incision was established on the affected side of the scrotum, then two Allis forceps hold the whole scrotal wall. (B) The scrotoscope was put into the tunica sac and the scrotal contents were inspected sequentially. (C) The location, appearance, size, margin of the epididymal mass were mainly observed. (D) Electrosurgical excision of the epididymal mass by plasma eletrosection was performed. (E) The wound got electrocoagulation to stop bleeding. $(F)$ The resected fragments of mass were retrieved. (G) A drainage strip was placed into the scrotum. $(\mathrm{H})$ The resected fragments of mass were sent for pathological examination. EM, epididymal mass. 
a.

Operating time

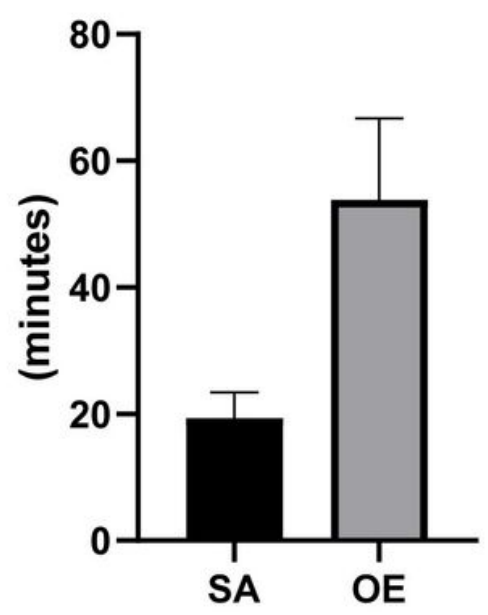

d.

Frequency of dressing changes

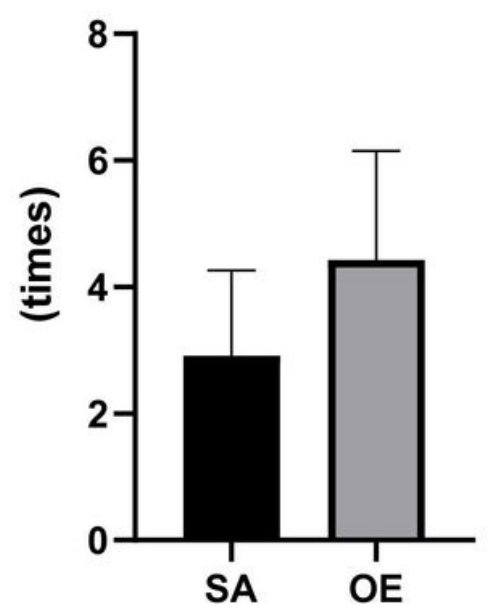

b.

Hemoglobin reduction

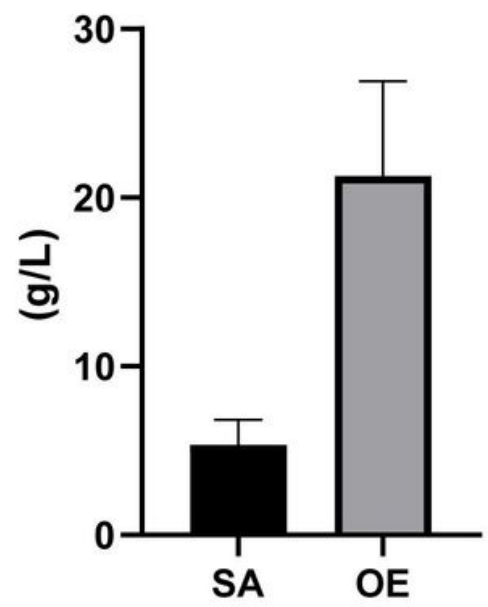

e.

Hospital stay

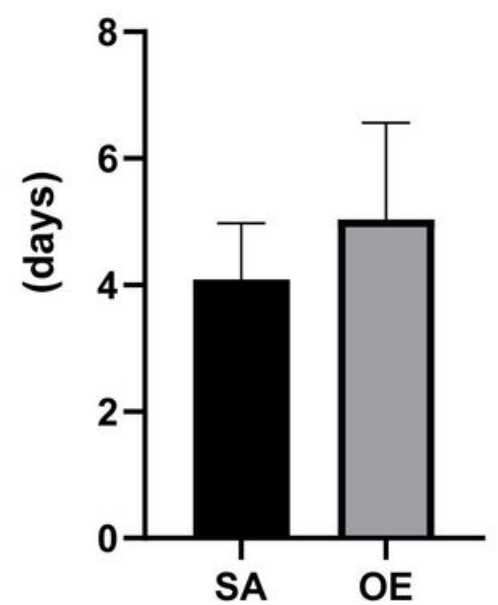

c.

Incision size

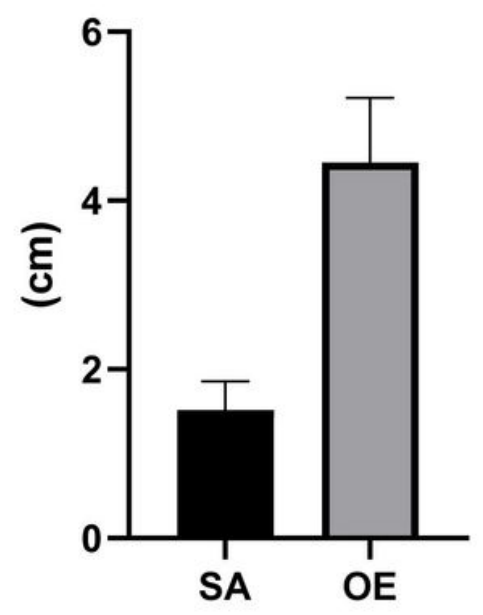

f.

\section{Satisfaction score}

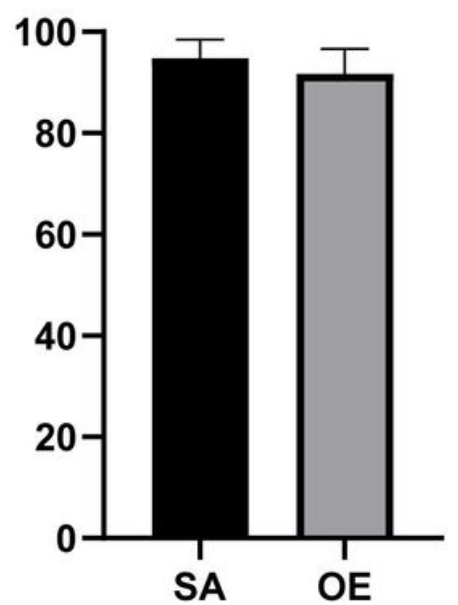

Figure 2

Intraoperative and Postoperative data. Scrotoscope-assisted excision (SA) showed less operating time, less blood loss, shorter length of incision, and less frequency of dressing changes. SA presented no significant advantage in the number of hospital stay. Besides, compared to open excision, SA also lead to a higher score in satisfaction (all P Value <.05). 